\title{
Learning interaction protocols by mimicking Understanding and reproducing human interactive behavior
}

\author{
Yasser Mohammad ${ }^{\mathrm{a}, * *}$, Toyoaki Nishida ${ }^{\mathrm{a}}$ \\ ${ }^{a}$ Kyoto University, Kyoto, Japan
}

\begin{abstract}
Understanding human interactive behavior is a key technology required for future robots. To achieve this goal, the robot should be able to recognize key patterns in human-human interactions.Moreover, the robot should be able to generate similar behaviors during its interaction with human partners. In this paper, an unsupervised system is proposed that allows the robot to build a generative model of the interaction protocol using interaction records. A system is evaluated in a guided navigation task and is shown to successfully learn the underlying interaction protocol.
\end{abstract}

(c) 2014 Elsevier Ltd. All rights reserved.

\section{Introduction}

Near-future robots are expected to be operated by untrained humans in office and home environments. To achieve this, these robots need to understand human behavior in order to be able to react appropriately to it both to accomplish the tasks they are designed to carry out and to increase the naturalness of human interaction with them.

Human behavior is complex and very difficult to model even in very simple situations. Consider, for example, how many ways a human can signal stop to another. This is not made any easier by cultural variabilities. For this reason, robots must rely on some form of autonomous learning to understand this behavior.

Several approaches to human behavior understanding have been proposed over the years and they can be divided into two major categories: discriminative methods that build a model of the behavior that can be used for prediction and generative models that allow the learner not only to predict human behavior but to produce similar behavior. Generative models are specially useful in robotics because the most distinctive feature of robotic agents is the ability to produce behavior. Even though it is not the case that human-like behavior is desirable for robots in all situations, in many cases it is desirable.

Learning from demonstrations can be considered a form of generative human behavior understanding because the learned

\footnotetext{
${ }^{* *}$ Corresponding author: Tel.: +81-80-42301024;

e-mail: yasser@ii.ist.i.kyoto-u.ac.jp (Yasser Mohammad)
}

pattern can be used to represent the behavior in a grounded way within the repertoire of the robot allowing it to predict future behavior as well as produce similar behaviors. Argall et al. (2009) provide a recent survey.

Most of the generative approaches (including learning by demonstration) focus on learning open loop behaviors (e.g. gestures, letters, etc (Tee et al., 2010)), object oriented behaviors (e.g. rotating around an object, navigation behavior, etc) or object manipulation behaviors (e.g. pouring liquid, moving chess pieces, etc). Little research was done in using similar techniques for understanding human's interaction oriented behavior in natural settings which is the focus of this work.

A basic assumption behind the proposed approach is that human interactive behavior can be understood as the execution of what we call interaction protocols. An interaction protocol can be either an explicit one that can be captured easily by a simple set of rules (e.g. responses to predefined gestures in an industrial workspace) or an implicit protocol that captures natural nonverbal interactions between people (e.g. gaze control during face to face encounters, rhythmic gestures accompanying speech, etc). An interaction protocol is defined by a number of roles (for partners). For example in a classroom there is a role for the instructor, and a role for students. Each role specify appropriate responses to actions of other roles involved in the protocol given the current state of the interaction and the environmental state. For example, an appropriate response to a student raising her hand in the class room might be for the instructor to allow here to ask a question.

Mohammad et al. (2010) proposed a hierarchical system for 
modeling and learning implicit interaction protocols. This approach produces interconnected interaction processes at multiple layers which makes it difficult to decode the details of the protocol. This reduces its applicability to understanding human behavior especially for the case of explicit interaction protocols. On the other hand, this approach works on the trajectory level assuming that reactions to partner's behavior is immediate which is not the case with explicit interaction protocols.

This paper extends and consolidates our previous work (Mohammad et al., 2009; Mohammad and Nishida, 2010) and provides a novel application of imitation learning to human interactive behavior. The first technical contribution of the proposed system is a novel algorithm for automatic segmentation of human behavior based on motif discovery. The second contribution of the system is a novel approach to controller generation based on piecewise linear motion primitives.

The main contributions of this paper are: the introduction of input dimension grouping of the action stream discussed (section 2.1), an improved algorithm for associating behaviors from multiple partners (section 2.2 and section 4.3) and an evaluation of alternatives for implementing the discovery phase of the proposed system (section 4.2).

The guided navigation scenario used as a running example in this work involves two agents (roles). The operator agent is a human that uses free hand gestures to instruct the actor agent (a robot in our case) to follow some path, avoid some obstacle, etc. The third agent is called the learner. The learner starts by watching some actor-operator interactions using a motion capture system and then uses unsupervised learning techniques to model both operator's gestures and actor's actions. It then builds a controller for each learned action and a detection mechanism for each learned gesture. The learner's goal is to be able to act in the actor's position.

The rest of the paper is organized as follows: Sections 2.1 to 2.4 details the algorithms used for the four phases of the proposed system. Section 4 provides a real-world experiment to evaluate the system. Section 5 discusses the findings of this experiment and compares the system with other generative understanding methods. The paper is then concluded.

\section{Proposed Learning Algorithm}

A bird's eye view of our approach is shown in Fig. 1. The proposed learning algorithm can be divided into four phases:

1. Discovery Phase: during which the robot discovers the action primitives associated with each role in the interaction.

2. Association Phase: during which the robot associates discovered actions from different interaction partners generating a probabilistic model that can be used for behaving and prediction.

3. Accumulation Phase: during which the probabilistic models learned in the association phase from multiple demonstrations are combined to form a generalized set of basic behaviors while combining their controllers.

4. Controller Generation Phase: during which the behavioral model learned in the association phase is converted into an actual controller to allow the robot to act in similar situations.

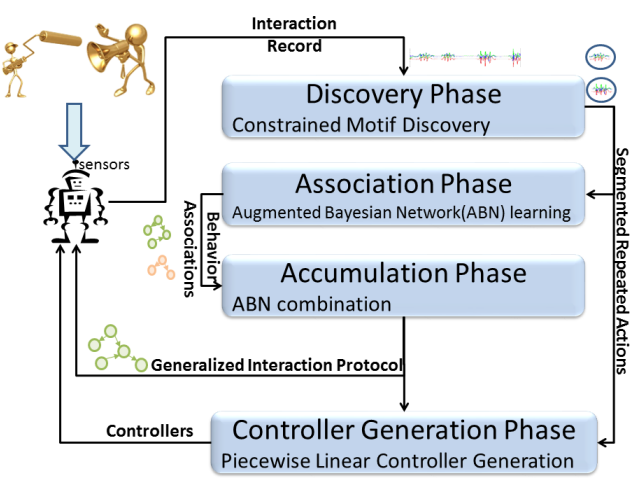

Fig. 1. Proposed four phases for interaction protocol learning showing the main algorithm of each phase as well as its inputs and outputs.

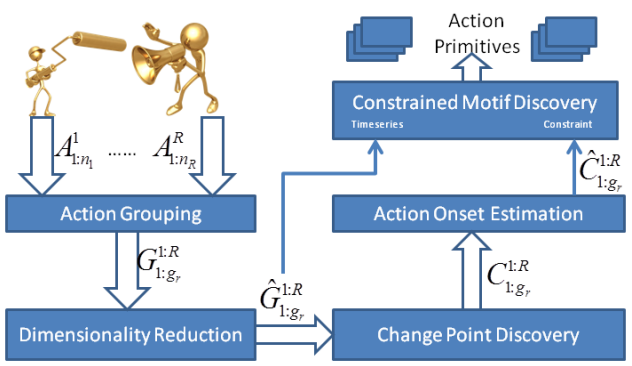

Fig. 2. The Discovery Phase. Input action records $(A)$ are grouped into subsets $(G)$ then the dimensionality of each subset is reduced to one $(\hat{G})$ and passed to the change point discovery algorithm and onset estimation algorithm to find probable locations of recurring patterns $(\hat{C})$ that are then passed to a constrained motif discovery algorithm to speed up the discovery of action primitives from the dimensionality reduced signal $(G)$

\subsection{Phase I: Discovery Phase}

The discovery phase is responsible for discovering the basic components of the observed behavior of the interaction partners (e.g. navigation primitives of the actor and commands/gestures of the operator in the guided navigation scenario). The main challenge in this phase is that the only available knowledge to the system are sensor readings of the interaction partners. The algorithm used in this phase does not distinguish gesture and navigation streams (which is desired not to depend on the specifics of them) and deals with the combination of them as a single stream of continuous multidimensional data called the action stream hereafter. An example for an input of this stage would be the relative orientation between the interaction partner or accelerometer data associated with hand movement of each partner, etc. Fig. 2 shows the computational steps involved in the discovery phase assuming $R$ roles in the interaction (e.g. $R=2$ for the guided navigation task and all diadic tasks that are the focus of this paper).

For the rest of this paper, we use the following terminology: $A_{i}^{x}$ stands for input dimension $i$ of the action stream corresponding to role $x$, where $1 \leq j \leq n_{x}$ and $G_{j}^{x}$ stands for the group $j$ of dimensions for role $x$ where $1 \leq j \leq g_{x}$ and $g_{x} \leq n_{x}$.

The action stream for each role $\left(A_{i}^{r}\right)$ is first divided into a disjoint set of groups $\left(G_{k}^{r}\right)$ to represent coherent sets of dimensions that are related to each other (e.g. sensors attached to each arm 
constitute a group). The dimensionality of each group of dimensions is reduced to one using Principle Components Analysis (PCA) to keep as much as possible of the variation in the motion while reducing the computational load on subsequent steps.

The next step is to find candidate locations of the actions in the action stream. For solving this problem we use a general change point detection algorithm called Robust Singular Spectrum Transform (RSST) to find locations around which there is an estimated change in the generating dynamics of the timeseries signaling the beginning or ending of an action primitive.

We then use the change scores found using RSST to calculate an estimation of the probability of finding an action primitive at every time step. The main idea is to utilize the assumption that there should be a causal relation between partners' behavior during an interaction. The technique used utilizes GangerCausality testing. Section 2.1.2 details this algorithm.

The final step is to use these probabilities to convert the continuous action stream into a concatenation of discrete behavioral primitives (called actions primitives from hereafter) that provide a more abstract representation of partners' behavior during the interaction. This can be achieved using a constrained motif discovery algorithm. Section 2.1.3 gives the definition of the constrained motif discovery problem.

Mohammad and Nishida (2010) used a similar approach but without grouping the action streams into disjoint sets. The grouping step proposed in this paper will be shown to improve the overall accuracy of the system by allowing it to discover more accurately action primitives that are executed using a subset of the demonstrator's DoFs (e.g. a gesture that uses a single hand).

\subsubsection{Discovering Change Points}

The first step in the discovery phase is to find the points in which the dynamics of every dimension of the action and command stream change. This is known as the change point discovery problem. To discover change points, we use the Robust Singular Spectrum Transform (RSST) method proposed by Mohammad and Nishida (2009b).

The essence of the RSST transform is to find for every point $x(i)$ the difference between a representation of the dynamics of the few points before it (i.e. $x(i-p): x(i))$ and the few points after it (i.e. $x(i+g): x(i+f)$ ). This difference is normalized to have a value between zero and one represents the change score at time step $t$. The dynamics of the points before and after the current point are represented using Hankel matrices. Singular Value Decomposition (SVD) is then used to find the singular values and vectors of the two Hankel matrices representing the past and the future of the timeseries.Only the first $l(t)$ left singular vectors of each Hankel matrix are kept and a weighted average of the angles between corresponding pairs of singular vectors are used to find an initial guess change score. This initial guess is then filtered to reduce the effect of white noise on the estimation. For more details and evaluations of this algorithm, please refer to Mohammad and Nishida (2009b).

\subsubsection{Action Onset Estimation}

The next step is to combine the change scores discovered in the previous step to generate an estimation of the locations of actions and commands to be used later as a constraint in the constrained motif discovery step (section 2.1.3). Because the response time of the actor is not in general known, it is necessary to estimate this response time in order to combine the change scores properly. Granger causality is a technique for determining whether one time series is useful in forecasting another (Gelper and Croux, 2007). In this paper we use it in a nonstandard way to determine the natural delay $\rho_{o p}$ between actions of different partners in the interaction being learned. The main idea is to find the delay that maximizes the GrangerCausality between the change scores of the two streams representing the change score of each partner's action stream. For the details of this algorithm, refer to Mohammad et al. (2009).

\subsubsection{Discovering Motifs}

The final step of the discovery phase is to find the recurring patterns in every dimension of the command and action streams. This is a motif discovery problem and is intensively studied in data mining literature.

To efficiently process the command and action streams, information from the change point detector should be explored to reduce the search space for patterns. The problem of discovering motifs in time series while utilizing a constraint about their probable locations is called Constrained Motif Discovery (CMD) and was defined by Mohammad and Nishida (2009a). In section 4 , we compare five alternative motif discovery algorithms for this phase.

\subsection{Phase II: Association Phase}

After completing the discovery phase, the learner has a list of action primitives for each role in the interaction. It now needs to associate these primitives to each other to build a generative model of the interaction. For example one action primitive may correspond to rotating clockwise while another primitive is a hand gesture signaling to rotate clockwise. This phase is responsible of discovering that that former is executed in response to the later. The main insight of our approach is that causation can be defined as the predictability of change in the effect given a change in the cause rather than the predictability of the effect itself given the cause itself.

We start by constructing for each action primitive $M_{i}^{r}$, an associated occurrence list $L_{i}^{r}$ that specifies the timesteps at which each occurrence of $M_{i}^{r}$ starts.

Our main assumption is that if action primitive $i$ for role $r 1$ causes the agent in role $r 2$ to execute the action primitive $j$ then $L_{j}^{r 2}$ will most of the time if not always has values that are $\tau_{i j}$ time-steps after $L_{i}^{r 1}$ where $\tau_{i j}$ is a constant representing the delay of the causation.

Since in the real world, many factors will affect the actual delay (add to this inaccuracies in the change point detection algorithm), we expect that in reality the delays between these action onset points can be approximated with a Gaussian distribution with a mean of $\hat{\tau_{i j}}$ where $\left|\hat{\tau_{i j}}-\tau_{i j}\right|<\epsilon$ for some small value $\epsilon$. The main idea of our algorithm is to check for the normality 
of the delays and to use the normality statistic as a measure of causality between the two processes.

We start with a causality graph with $n$ nodes representing the processes and no edges. Second, for each pair of action onset location vectors $\left(L_{i}^{r 1}\right.$ and $L_{j}^{r 2}$ in order); we find the list of all delays $\left(\left\{\tau_{i j}{ }^{k}\right\}\right)$. Notice that in general (if no causal loops exist) the sets $\left\{\tau_{i j}{ }^{k}\right\}$ has nothing to do with $\left\{\tau_{j i}{ }^{k}\right\}$. This is the reason that our graph will be directed.

To guard against inaccuracies in action onset discovery we remove from the set $\left\{\tau_{i j}{ }^{k}\right\}$ all points that are more than 4 standard deviations from its median assuming that these points are outliers. Removal of outliers serves also to allow the system to work if the change happens with a probability less than one (but is still high enough to be detected).

We then calculate a causality score from set $\left\{\tau_{i j}{ }^{k}\right\}$ using its mean $\left(\mu_{i j}\right)$ and standard deviation $\left(\sigma_{i j}\right)$ (after removing the outliers as explained before) by:

$$
\text { score }=1-\exp \left(-\sigma_{i j} / \mu_{i j}\right)
$$

The larger this score is, the more probable is it that there is a causal relation between processes $i$ and $j$. To construct the causality graph we accept the causal relation if this score was over some predefined threshold. In the causality graph we add an edge from $M_{i}^{r 1}$ to $M_{j}^{r 2}$ and associate with it the mean and variance of the delays $\left(\mu_{i j}, \sigma_{i j}\right)$ calculated from $\left\{\tau_{i j}{ }^{k}\right\}$. It is also possible to add to this edge's label a confidence measure by dividing the number of times $L_{j}$ has a change after $\mu_{i j} \pm \delta$ from a change in $L_{j}$ to the number of changes of process $i\left(\left|L_{i}\right|\right)$. This confidence measure characterizes the predicting power of this causal relationship. We write this information as:

$$
i \stackrel{\mu_{i j}, \sigma_{i j}, c_{i j}}{\longrightarrow} j
$$

After completing this operation for all $L_{i}^{r 1}$ and $L_{j}^{r 2}$ pairs (where $r 1 \neq r 2$ ), we have a directed graph that represents the causal relationships between the series involved in the form of a Bayesian Network with the edges augmented with information about explicit delays. We call this graph the Augmented Bayesian Network (ABN) hereafter.

\subsection{Phase III: Controller Generation Phase}

Each action primitive that was learned in the discovery phase represents a basic motion/action but in the sensory space of the learner. For example, in the navigation task, gestures are being learned as 3D motions and navigation primitives are being learned in the 2D space in which the actor is moving. The learner cannot directly use this information to control its actuators and it needs to learn a controller for each of these primitives that is capable of executing it using the body of the learner.

Learning these controllers can be thought of as a standard Learning from Demonstrations (LfD) problem. For the navigation action, controller generation is achieved in two sub-stages. Firstly, reinforcement learning is used to allow the learner to generate its basic motion primitives related to the action stream dimensions. This is called the motor Babbling stage. Once these primitives are learned, the robot starts to generate controllers as sequences of primitives that generate the required pattern in the action dimensions. This is the second stage and is called Piecewise Linear Controller Generation (PLCG).

\subsubsection{Motor Babbling}

During motor babbling, the robot builds a repertoire of motor primitives related to the action dimensions that will be used for controller generation. These primitives are not related to the motifs learned during the discovery phase but are simpler and provide building blocks to these motifs' implementation. These primitives are constituted from two functions for every dimension: one to increase and the other to decrease that dimension with minimal impact on the value of others. Section 2.3.2 shows how these functions can be used to simplify the development of controllers for the learned actions.

To reduce the risks on the learner robot during this phase, a simulator was used. The robot starts in a predefined initial state (specific values for $A$ ) and tries random motion actions (commands to its motors $C$ ) keeping track to the changes happening to each of its action dimensions. This training data is then used to learn two functions for every dimension called the increment and decrement functions. These two functions take as input the current action stream state and produce motor commands according to the following rules: The increment function of dimension $i$ (hereafter $F_{i}^{+}$) increases the value of this dimension by some rate $\left(\delta_{i}\right)$ while keeping the change in other dimensions as small as possible. The decrement function (hereafter $F_{i}^{-}$) reduces the value of this dimension by some rate $\left(\delta_{i}\right)$ while keeping the change in other dimensions as small as possible.

Thus $F_{i}^{+}$and $F_{i}^{-}$both solve the following constrained optimization problem with positive and negative $\delta_{i} \mathrm{~s}$ :

Minimize:

$$
\sum_{\substack{j=1: n_{a} \\ i \neq j}}\left(a_{j}(n+1)-a_{j}(n)\right)^{2}
$$

Constraints:

$$
\begin{aligned}
& \text { for } 1 \leq i<n_{a} \\
& \left|a_{i}(n+1)-a_{i}(n)\right| \geq \delta_{i}^{-} \\
& \left|a_{i}(n+1)-a_{i}(n)\right|<\delta_{i}^{+}
\end{aligned}
$$

Control Variables:

$$
C \equiv\left[\Delta m^{+}(n), \Delta m^{-}(n)\right]
$$

where $a_{i}(n)$ is the $n$ 'th sample of the $i$ 'th action dimension, $\Delta m^{+}$ is the sum of the two commands given to the motors and is proportional to the speed, $\Delta m^{-}$is the difference between these two commands and is proportional to the rotation angle (differential drive arrangement), $\delta_{i}^{+}$is the upper limit on the required rate of change, and $\delta_{i}^{-}$is the lower limit. The pair $\Delta m^{+}$and $\Delta m^{-}$ constitute the command sent to the motors $C$.

The problem is formulated as a Markov Decision Process (MDP) and is solved using standard Q-Learning with the aid of a simulator that can produce $A(n+1)$ given $A(n)$ and $C(n)$. The resulting $F_{i}^{+}$and $F_{i}^{-}$can now represent a straight 
line in the action dimension $i$ with an approximate slope of $\delta_{i}=\left(\delta_{i}^{-}+\delta_{i}^{+}\right) / 2$. These two functions thus serve to linearize the relation between the action dimensions and motor commands and in the same time decouple different action dimensions.

\subsubsection{Piecewise Linear Controller Generation}

The second and final step is generating the required controllers given the $\mathrm{ABN}$ and the $F_{i}^{+}, F_{i}^{-}$functions. Firstly, the mean pattern attached with the node is approximated by a piecewise linear time series using the SWAB algorithm (Keogh et al., 2001). Secondly, the slope of each line segment is calculated for every action dimension included in the pattern and $F_{i}^{+}, F_{i}^{-}$ functions are used to generate a controller for each of these dimensions. This results in a sequence of $F_{i}^{+}$and $F_{i}^{-}$calls that produce the required pattern.

This is an open loop controller and in real world can cause large errors. To correct for that, the difference between the actual action stream (state) as perceived by the robot and the piecewise mean approximation $(\hat{m})$ is calculated at a frequency of $10 \mathrm{~Hz}$ and functions $F_{i}^{+}$and $F_{i}^{-}$are then used to correct for the error with a rate equal to $\pm \zeta \delta_{i}$ where $\zeta>0$ and $\delta_{i}$ is the slope of the current linear segment of $\hat{m}$. In the experiment reported in this paper we used $\zeta=1$. This closes the loop and produces the final closed loop controller.

This simple approach did work in the navigation case because the motifs in the action stream were mostly piecewise linear. In more complex motif forms (e.g. the gestures in the gesture stream), the applicability of this technique will be limited and further research is required to allow the learner to generate more complex nonlinear controllers.

\subsection{Phase IV: Accumulation Phase}

By the end of the controller generation phase, the learner robot will be able to replace the actor in the guided navigation scenario. Nevertheless, new human partners (operators) will tend to use different gestures or may use some already learned gestures with a different meaning. The robot then needs a mechanism to improve the learned protocol (i.e. the ABN) either by watching new interactions or during its own engagement in guided navigation. This section describes a simple mechanism for achieving this goal. The input in this case is two ABNs learned from two different interaction and the output is a single ABN that combines them.

To combine two different $\mathrm{ABNs}$ we need to discover nodes in the two ABNs that represent the same action primitives and combine them while keeping nodes of every $\mathrm{ABN}$ that have no counterparts in the other one. The simplest approach is to compare patterns stored in each node from the two ABNs and combine any two nodes belonging to the same role if the distance between their parameters is less than some predefined threshold. This approach is expected to have limited success because it does not take into account the relation of every node to other nodes in the ABN which prevents it from using the full information embedded in the ABNs in solving the association problem between nodes. In the same time, it is hard to decide the value for the threshold that will give good false rejection/false acceptance balance.
Mohammad and Nishida (2010) proposed a different approach that try to utilize more information from the two ABNs. We use the same approach here. The algorithm starts by compiling two lists of action nodes for every role from the two ABNs (namely $A N^{1}$ and $A N^{2}$ ). For every member of $A N^{1}$ (called $a n_{i}^{1}$ ), the motif mean $\left(m_{i}^{1}\right)$ is compared with every other motif mean in the same ABN $\left(m_{j}^{1}\right)$ using Dynamic Time Wrapping (DTW) and the minimum distance is selected as the similarity threshold of this node $\tau_{i}$ :

$$
\tau_{i}=\min \left(d_{D T W}\left(m_{i}^{1}, m_{k}^{1}\right)\right)
$$

where $1 \leq k \leq n_{A}^{1}, k \neq i$, and $n_{A}^{1}$ is the number of action nodes in $A N^{1}$.

The second step is to compare the mean of node $i$ with every other node in the second list $A N^{2}$ and a link $l a_{1 i}^{2 j}$ is created between $a n_{i}^{1}$ and $a n_{j}^{2}$ iff: $d_{D T W}\left(m_{i}^{1}, m_{j}^{2}\right)<\tau_{i}$ and $\left(d_{D T W}\left(m_{i}^{1}, m_{j}^{2}\right)-d_{D T W}\left(m_{i}^{1}, m_{k}^{2}\right)\right)<\eta \tau_{i}$ for $1 \leq k \leq n_{A}^{2}$ and $k \neq j$ for some value of $\eta$ greater than zero. We select $\eta$ to equal 0.25 for all our experiments. If two or more nodes in $A N^{2}$ satisfy these two conditions, a link is created between $a n_{i}^{1}$ and each of them. Each link had a value equal to the DTW distance between the means of the two nodes it links.

The final step is to remove all the conflicts in the two link lists to have at most one node in the second $\mathrm{ABN}$ connected to any node in the first ABN. For every link we calculate a link competence index (LCI) that evaluates the match between the two ABNs if this link was kept as follows:

$$
\begin{aligned}
& \operatorname{LCI}\left(l a_{1 j}^{2 i}\right)=\frac{1}{v\left(l a_{1 j}^{2 i}\right)}+\lambda_{a} \sum_{\substack{g n_{l}^{2} \in \operatorname{Par}\left(a n_{i}^{2}\right) \\
g n_{k}^{1} \in \operatorname{Par}\left(a n_{j}^{1}\right)}} \operatorname{LCI}\left(\lg _{1 k}^{2 l}\right) \\
& \operatorname{LCI}\left(\lg _{1 j}^{2 i}\right)=\frac{1}{v\left(\lg _{1 j}^{2 i}\right)}+\lambda_{g} \sum_{\substack{g n_{i}^{2} \in \operatorname{Par}\left(\operatorname{an}_{l}^{2}\right) \\
g n_{j}^{1} \in \operatorname{Par}\left(\operatorname{an}_{k}^{1}\right)}} \operatorname{LCI}\left(l a_{1 k}^{2 l}\right)
\end{aligned}
$$

where $\operatorname{Par}(n)$ is the set of all parents to node $n$. These equations constitute a set of $n_{l a}+n_{g a}$ equations in the same number of variables and can be solved using a simple iterative approach similar to the value iteration for solving MDPs.

A larger LCI means that not only the primitive nodes connected by the link are similar but also their parent nodes are similar as well. After the LCI is calculated for every link, the link with highest LCI fanning out from any node is kept and the rest are discarded.

After resolving all conflicts, the nodes in the two ABNs that are still linked are combined their stored pattern is re-generated from the full set of motif occurrences used when creating the two ABNs.

Combining nodes from two ABNs does not affect the edges except if it caused two nodes to be connected by more than one edge in the final ABN. In this case, the mean and variance of the delay associated with the final edge are calculated from the 
mean, variance, and number of occurrences in the two combined edges.

The main advantage of this approach is that it utilizes information from the whole $\mathrm{ABN}$ in determining the similarity between nodes. Another advantage is that the thresholds are adjusted per node and are determined automatically from the data.

\section{Building Block Alternatives}

Fig. 1 gives a general architecture with several building blocks and the system reported in the previous section provides but a single possible instantiation of this architecture. It is possible though to modify the system by replacing any of its building blocks (e.g. motif discovery algorithm, change point discovery algorithm, etc) with other variants. In this section, we discuss our rationale for choosing the specific algorithm used for each building block.

Change point discovery is the first building block in our approach. Research in change point (CP) discovery problem has resulted in many techniques including CUMSUM, autoregressive modeling (Gombay, 2008), Discrete Cosine Transform, and Singular Spectrum Transform (SST) (Idé and Inoue, 2005). Most of these methods with the exception of SST either discover a single kind of change (e.g. CUMSUM discovers only mean shifts), require ad-hoc tuning for every time series (e.g. wavelet analysis), or assumes a restricted generation process (e.g. Gaussian mixtures). RSST had the extra advantage of achieving higher noise resistance compared with SST. This work utilized RSST because of its generality which allowed us to use it for gesture and motion streams in the guided navigation scenario.

Motif discovery is the main building block in our approach. Research in motif discovery have led to many techniques including the PROJECTIONS algorithm (Chiu et al., 2003), PERUSE (Oates, 2002), Gemoda (Jensen et al., 2006) among many others. In section 4.2, we compare five different motif discovery algorithms for our application. Even though different algorithms showed different strengths (see Table 1), MK++ (see section 4.2) provides an attractive alternative for our application because of its ability to discover more of the basic actions embedded in the action stream.

Another building block of the system is the algorithm used for associating learned actions. Mohammad and Nishida (2010) used a simple association algorithm to build a probabilistic network of gestures and their associated actions. This method relied heavily on the distinction between actions and gestures which makes it inappropriate for generalization beyond the guided navigation scenario. Moreover this method required the specification of a hard-to-specify consistency threshold (the minimum number of times a gesture is followed by an action). In this paper, we handled both of these problems by utilizing a change causality discovery algorithm for the association phase 2.2.

The final building block of our system is the controller generator which can be thought of as a Learning from Demonstration (LfD) problem. Recently, two main approaches to LfD can be identified in the literature. The first approach is based on probabilistic modeling of the demonstration in a way that facilitates regeneration of the learned behavior while the second uses nonlinear dynamical systems to represent a parameterized version of the behavior.

Several probabilistic techniques have been used in LfD including Hidden Markov Models (HMM), Gaussian Processes (GP) and Gaussian Mixture Models (GMM). A technique of special importance in this category that found a widespread utilization is the Gaussian Mixture Modeling/Gaussian Mixture Regression (GMM/GMR) approach in which the example demonstrations are time-wrapped using dynamic programming to have the same length and the resulting data is modeled by a GMM. For behavior generation, GMR is used to recover a smooth motion trajectory.

Nonlinear Dynamical systems are also widely used to model demonstrations. A representative example of this approach is the Discrete Motor Primitives (DMP) approach (Ijspeert et al., 2002) which uses a single demonstration. The main idea is to model the motion by transforming a canonical system of differential equations with well-defined attractor properties using a nonlinear set of kernels to produce a behavior similar to the demonstrated behavior while being guaranteed to approach the goal state of the motion.

Both GMM/GMR and DMP provide attractive features in terms of encoding-utility and in our running example they are both applicable to gesture learning. Nevertheless, actual control of the robot requires a solution to the correspondence problem (recovering motor commands for the learner that are appropriate to generate the perceived behavior of the demonstrator). A solution to the correspondence problem will pre-suppose knowledge of the forward kinematics/dynamics of the learner which we do not assume in this work. This is why we needed the motor babbling step. Moreover, both of these approaches generate smooth trajectories which is an advantage for gesture learning but not for navigation behavior using differential drive controllers where motion primitives are usually discontinuous.

\section{Evaluation}

This section presents a feasibility study to assess the applicability of the proposed approach in learning guided navigation by untrained novice users.

\subsection{Experimental Setup}

Eighteen subjects were recruited for this experiment (10 males and 8 females) with ages ranging from 18 to 31 . All subjects were university students and they had no professional experience in operating robots. 15 of the subjects never operated a robot before. The goal of the experiment was to compare the performance of the learner robot in performing the actor role in guided navigation under three settings:

- WOZ: Wizard of OZ arrangement in which the robot is remotely controlled by a hidden human operator. The hidden operator watches the gestures of the subject and issues motion commands to the robot 
- Per-Participant Learner: The robot controller is developed using the first three stages of our approach (without accumulation) from a single interaction with the subject and then used as the actor with the same subject.

- Accumulating Learner: The robot controller is developed using the four stages of our approach and then tested with a subject it never encountered before.

After completing a background evaluation questionnaire and attaching PhaseSpace motion capture markers and B-PACK (Ohmura et al., 2006) wireless accelerometers, every subject conducted four sessions. In every session the goal of the subject was to instruct a robot to follow a path drawn in the ground using free hand gestures. The subjects were told that both the accuracy of following the path and the total time needed to complete it were used to evaluate the performance of the robot. Moreover, the subjects were informed that every session uses a different robot controller and that they will be asked to compare them.

In the first session, the robot was WOZ controlled. This session served two purposes: Firstly, it allowed the learner to collect training data in the form of $G$ and $N$ streams. Secondly, it allowed the subject to get used to controlling the robot using hand gesture. After each session, the subject filled a questionnaire to evaluate the performance of the robot during this session. Because the learning robot did not have any access to the behavior of the WOZ operator (only $G$ and $N$ ), the learning system is still unsupervised and the WOZ operator can be considered as a part of the actor. The main reason for using this arrangement is not to pre-program the actor with fixed action/gesture relations that can be easily learned. The WOZ operator had the choice between seven motion primitives (i.e. rotate left, rotate right, come here, go away, move forward, and stop). This means that the causal structure of the session is known with seven primitive gestures having outgoing links to seven primitive actions in bipartite structure. Moreover, the start and ending times of each gesture and the corresponding action were logged to provide a ground truth for comparing different discovery phase algorithm combinations.

While the subject was filling the first session questionnaire, the Per-Participant learner applied the first 3 stages of the proposed approach to generate an $\mathrm{ABN}$ representing the interaction protocol used by this user.

The accumulating learner combined this $\mathrm{ABN}$ with its current $\mathrm{ABN}$ but the combined $\mathrm{ABN}$ will not be used with this participant but the next one. This ensures that the accumulating learner did not use any information from the user it was tested with in any of the 17 trials (The first participant did not interact with the accumulating learner because there was no ABN available other than the one created using this participant's data).

The final three sessions were conducted in the three conditions described before (WOZ, Per-Participant Learner, and Accumulating Learner). The order of these sessions was randomly shuffled to counter any ordering effects.

To test the generalization of the learning model, the path used in the first session during training was different from the path used in the final three test sessions. If the subject was not able to get the robot to the goal within 20 minutes the session was considered a failure and was aborted.

In each post-session questionnaire, the subjects evaluated the robot they interacted with using a scale from 1 to 7 in the following dimensions:

1. Ease of guiding the robot.

2. Ability of the robot to understand gestures.

3. Attentiveness of the robot.

4. Accuracy of the robot in following the gestures.

5. Naturalness of robot's behavior.

After all the sessions, the subject selected one of the last three robot controllers as her/his preferred actor.

The sessions were conducted in six days with three sessions every day. The same experimenter acted as the hidden operator in all training and WOZ sessions.

The inputs to the learning robot are two multidimensional time series:

1. Gesture stream $(G)$ representing the motion of the guiding human. In this work we use wireless accelerometers called B-PACK (Ohmura et al., 2006). Two accelerometers attached to the middle finger tips of the operator's two hands which results in a six dimensional gesture stream.

2. Navigation Stream $(N)$ representing the motion of the guided human/robot. In this work we use a motion capture system called PhaseSpace to measure the location of the actor in the arena.

To calculate the navigation stream we first capture the position of the actor and the operator using the PhaseSpace motion capture system which can determine the 3D position of a set of markers. Six markers where attached to the head of the operator and six markers around the actor. This constitutes a 36 dimensional raw data stream. The 2D absolute positions of the operator and actor in the floor were calculated from this raw data by first estimating the center of each agent using each available marker attached to it and averaging to reduce the effect of noise and missing marker positions. This was necessary because of the high rate of missing data from the motion capture system.

The navigation stream $N$ was calculated from these positions by calculating the following:

- 2D distance and angle $\left(r_{o}, \theta_{o}\right)$ between the actor and the operator in the operator's coordinate system.

- 2D position of the actor in the plan of motion $\left(x_{a}, y_{a}\right)$ in Cartesian coordinates.

- Orientation of the actor in the plan relative to the direction of its starting point $\left(\theta_{a}\right)$.

These features constitute the 5-dimensional navigation stream $(N)$. These features were selected to cover both operator-centric and robot-centric direction and orientation. The goal of the learner in the guided navigation scenario is to use only $N$ and $G$ with no prior knowledge to learn how to act in the actor's role and then to actually replace the actor in future sessions. 


\subsection{Evaluating the Discovery Phase}

The heart of the discovery phase (section 2.1) is the constrained motif discovery which is responsible of finding the final action primitives for each role. We used the ground truth data from the WOZ operated sessions (36 in total).

Five motif discovery algorithms were compared: PROJECTIONS, DGCMD, Catalano's algorithm, MK++, and MOEN. PROJECTIONS algorithm symbolizes the input stream and uses location sensitive hash functions to estimate the similarity between patterns (Tompa and Buhler, 2001). DGCMD was used by Mohammad et al. (2009) for discovering gestures and navigation motion primitives and Catalano's algorithm uses random sampling of long enough subsequences and multiple distance comparisons within these candidate subsequences for motif discovery (Catalano et al., 2006). MK++ is an extension of the MK algorithm proposed by Mueen et al. (2009) to discover the most similar pair of subsequences in a timeseries (Mohammad and Nishida, 2014). MOEN is another exact motif discovery algorithm that was proposed recently by Mueen (2013).

These algorithms were selected to cover the current three main trends in motif discovery. MOEN and MK++ represent exact motif discovery algorithms. PROJECTIONS represent discretizing algorithms. DGCMD/Catalano's algorithm represent approximate motif discovery algorithms that utilize change point information to speedup the discovery process.

Comparing motif discovery algorithms is not trivial due to possible shifts in discovered motif boundaries. For our application, false negatives are much more important than false positives because subsequent stages can get rid of false positive primitives by associating them with nothing. Moreover, complete primitive coverage is essential (either in a single or multiple discovered motifs) to give the controller generation phase a chance to generate a useful controller. Table 1 shows the average results after applying the five algorithms to both gesture and navigation streams of the $36 \mathrm{WOZ}$ operated sessions for which accurate ground truth is available. A false negative means that a primitive (either a gesture or navigation primitive) was not found at all by the algorithm. A false positive means that a pattern that does not correspond to a primitive was found. The percentage covered applies only to discovered primitives and measures that fraction of the primitive length that was found, while the percentage extra measures the extra points (if any) that are appended to discovered primitives. The first finding is that the grouping step proposed in this paper reduced the number of false negatives for all algorithms (except PROJECTIONS for which it had no effect). In most cases, it also increased the number of false positives but this is not a critical problem because the association phase will be able to remove these fictitious primitives when no causal connection between them and the behavior of the other partner is discovered.

The algorithms that led to the minimum number of false negatives (with grouping) was $\mathrm{MK}++(F N=0.0794)$ followed by $\operatorname{MOEN}(F N=0.127)$ and DGCMD $(F N=0.1468)$. PROJECTIONS and Catalno's algorithms achieved much worse in this dimension. DGCMD covered the highest percentage of discovered primitives $(C=87 \%)$ on average and MOEN had the lowest amount of extra-noise appended to discovered patterns $(E=7 \%)$.

\subsection{Evaluating the Association Phase}

To evaluate the proposed algorithm for the association phase (section 2.2), we compared the learned network using this algorithm and the method proposed by Mohammad and Nishida (2010) (called simple counting from now on) on the data from the $36 \mathrm{WOZ}$ operated sessions. The evaluation criterion was the similarity between the discovered network and the ground truth causal structure of the interaction which is known to be a bipartite graph with seven gesture nodes and seven navigation action nodes. Every gesture node should have a single outbound link to a single action node.

Since the primitive (e.g. a gesture) may be learned partially as a set of two or more motifs, the nodes in the learned graph cannot be directly corresponded to the nodes in the ground-truth bipartite graph. To resolve this problem we combined all the nodes corresponding to partial discoveries of the same primitive in a single node with all the links inbound and outbound from it connected to this aggregated node. The resulting graph is called the aggregated graph.

Since we know the gesture/navigation action corresponding to each node in the aggregated graph (if any), it is straightforward to calculate the number of correct links and the number of incorrect links in the graph by comparing it with the groundtruth graph described earlier. Again, we can use the number of false positive and the number of false negative links in the graph for each of the $36 \mathrm{WOZ}$ operated sessions for both algorithms. Success is defined by the links in the aggregated graph matching links in the bipartite ground truth graph.

The proposed algorithm has a false positive rate of $8 \%$ compared with $11 \%$ for simple counting with a false negative rate of $4.3 \%$ compared with $3.9 \%$ for simple counting. This shows that the proposed algorithm could better reject wrong associations while keeping roughly the same sensitivity achieved by simple counting. This is despite the fact that the proposed algorithm does not explicitly assume that the direction of causation is from gestures to actions (as is the case in simple counting) which makes it more appropriate for generalization to more complicated interaction protocols that involve turn-taking (e.g. gaze control, back-channeling during speech, etc).

\subsection{Evaluating the Complete System}

For the sake of completeness, this section briefly discusses the findings for complete system evaluation. Mohammad and Nishida (2010) provide a more detailed account of these results. Fig. 3 shows the average score of the three controllers over the days of the experiment. It is clear that the WOZ operator had good performance in the whole experiment which is expected.

The first interesting finding from the figure is that the perparticipant learner could achieve indistinguishable behavior from the human operator in the WOZ operator after the single training session. The discovery and association algorithms used in this online experiment were DGCMD and simple counting. This suggests that the performance can be further improved by utilizing the $\mathrm{MK}++$ algorithm for the discovery phase and 
Table 1. Comparison between different motif discovery algorithms for the discovery phase.

\begin{tabular}{|c|c|c|c|c|c|c|c|c|}
\hline Algorithm & $\begin{array}{r}\text { False Ne } \\
\text { Grouping }\end{array}$ & $\begin{array}{l}\text { gatives (FN) } \\
\text { No-Grouping }\end{array}$ & $\begin{array}{r}\text { False Pc } \\
\text { Grouping }\end{array}$ & $\begin{array}{l}\text { sitives (FP) } \\
\text { No-Grouping }\end{array}$ & $\begin{array}{l}\text { Percentag } \\
\text { Grouping }\end{array}$ & $\begin{array}{l}\text { Covered (C) } \\
\text { No-Grouping }\end{array}$ & $\begin{array}{r}\text { Percenta } \\
\text { Grouping }\end{array}$ & $\begin{array}{l}\text { age Extra (E) } \\
\text { No-Grouping }\end{array}$ \\
\hline MK++ & 0.0794 & 0.1389 & 0.4246 & 0.4167 & $85 \%$ & $82 \%$ & $14 \%$ & $18 \%$ \\
\hline MOEN & 0.1270 & 0.2024 & 0.2123 & 0.3750 & $55 \%$ & $61 \%$ & $7 \%$ & $10 \%$ \\
\hline DGCMD & 0.1468 & 0.1627 & 0.3413 & 0.3988 & $87 \%$ & $79 \%$ & $16 \%$ & $26 \%$ \\
\hline PROJECTIONS & 0.3095 & 0.4008 & 0.1290 & 0.1944 & $41 \%$ & $38 \%$ & $10 \%$ & $21 \%$ \\
\hline Catalano & 0.6627 & 0.7222 & 0.4028 & 0.3611 & $14 \%$ & $27 \%$ & $31 \%$ & $20 \%$ \\
\hline
\end{tabular}

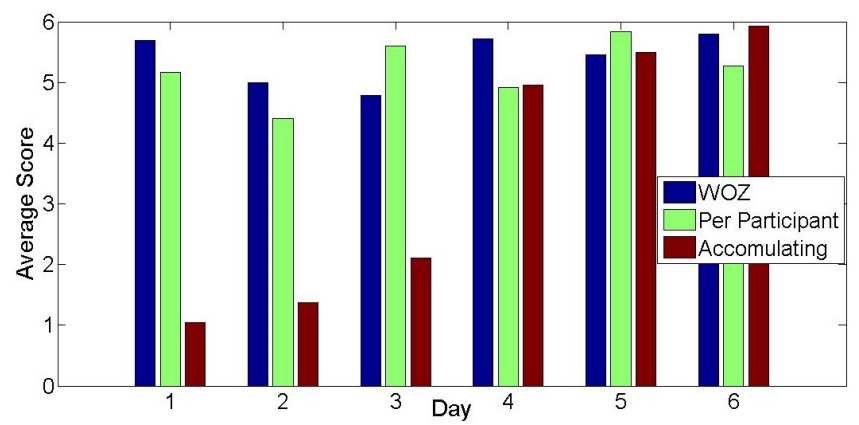

Fig. 3. Average subjective scores assigned by the 18 participants over the six days of the experiment.

the association phase algorithm proposed in this paper. Online evaluation of this combination is left for future research.

Another finding from Fig. 3 is that the performance of the accumulating learner could achieve the same level of performance as the per-participant learner (and the WOZ operator) by the fourth day slightly outperforming both of them by the last day. This shows that the proposed accumulation phase could effectively combine the information learned from different sessions.

An important feature of the accumulating learner's performance is that it did not improve linearly or smoothly. There was a sudden improvement in performance in the fourth day after a period of nearly no improvement in the first three days. One explanation of this phenomenon is that the learner needed to interact with several participants in order to see the different kinds of gestures used for each navigation command. The performance improved in the second half of the experimental days once the robot was able to collect enough examples of gestures in the first three days.

To quantify the difference between the two learners objectively, we calculated the Pearson correlation between the average score in the three conditions and the participant number. The accumulating learner showed a correlation of $81.94 \%$ with the participant order and the p-value was 0.000032 showing a statistically significant correlation. The WOZ and perparticipant learner showed no statistically significant correlation with the participant order.

The participants selected the WOZ operated robot as their preferred robot eight times, the per-participant learner seven times and the accumulating learner three times (all in the last two days). Again, the effect of time on the evaluation of the accumulating learner was evident. In the first two days no one preferred the accumulating learner, while in the last two days half of the participants preferred it even over the WOZ oper- ated robot. The WOZ operated robot and per-participant learner were each preferred three times in the first two days and the perparticipant learner was preferred two times (compared to one for the WOZ operated robot) in the last two days.

\section{Discussion and Related Work}

Several discriminative approaches to human behavior understanding have been proposed over the years. For example Çeliktutan et al. (2012) proposed a method based on graph matching for human action recognition in video. Chung and Liu (2008) used a Hierarchical Context Hidden Markov Model (HC-HMM) for behavior understanding from video streams in a nursing center.

Discriminative approaches can provide acceptably accurate models of human behavior in limited environments but they are not applicable to robotics where the goals in not only predicting behavior but generating it. Even on the level of understanding, generative models have an advantage because the ability to generate similar behavior makes it possible to test the system in situations that it never encountered.

Most generative approaches to modeling human behavior focused on situations in which a single human is modeled. For example, Calinon et al. (2012) proposed a technique that combines dynamical systems for modeling and Gaussian Gesture Mixtures/Gaussian Mixture Regression for learning motion primitives from demonstrations. The approach shows impressive imitation abilities and does not suffer from the problems of discriminative approaches but it relies on pre-segmented demonstrations which limits its applicability to situations in which the demonstrator is actively teaching the skill to the learner.

Kulic et al. (2007) proposed a method for incremental learning of human body motion primitives from observation. Stochastic segmentation is used to first segment the motion stream into segments. The segments are then clustered incrementally and organized into two structures: A tree structure that represents the behavior at increasingly more abstract level of abstraction and a graph structure that relate learned primitives to each other. The main differences between this system and the proposed approach lie in the following: Firstly, our approach learns interaction protocols rather than single-agent actions. Secondly, the proposed approach relies on change point discovery followed by constrained motif discovery rather than the incremental segmentation algorithm used by Kulic et al. (2007). 
One limitation of our approach is that it operates in an offline mode where the learner just watches that interaction and builds its own learned protocol from the interaction record. This is not how humans learn in most cases. We usually learn by engaging in the task to be learned and improve our abilities gradually by utilizing feedback from the environment and mentors. Designing an appropriate adaptation mechanism for the agent is a direction for future research.

Another limitation of the proposed system is that it assumes that the interaction protocol runs on a single time resolution at a single abstraction level which is encoded in the causal structure of the interaction. Natural human interactions involve hierarchical interaction protocols. In the proposed architecture, a hierarchical system can be learned by treating the activation of learned primitives as inputs to a higher-level learner, yet this is beyond the scope of the current work.

\section{Conclusion}

This paper considered learning a generative model of human interactive behavior. The proposed system takes as input a record of unsegmented behavior of all interacting partners and has four phases. In the first phase, the input streams are segmented into basic actions using change point discovery followed by constrained motif discovery where the constraints are generated by maximizing the causal influence of the partner's behavior on each other. The second phase builds a probabilistic model of the causal interaction between the behaviors of the partners. The third phase generates a controller for each basic action that allows the learner to execute this action. The final phase allows the learner to combine probabilistic networks learned from multiple interactions in order to build a generalized version of the interaction situation. A guided navigation scenario was used for evaluating the predictive and generative aspects of the system in two real world experiments. Evaluation of experimental results show that the proposed approach is applicable in learning interactive human behavior.

\section{Acknowledgments}

This research is supported by the Center of Innovation Program from Japan Science and Technology Agency, JST.

\section{References}

Argall, B.D., Chernova, S., Veloso, M., Browning, B., 2009. A survey of robot learning from demonstration. Robotics and autonomous systems 57, 469483.

Calinon, S., Li, Z., Alizadeh, T., Tsagarakis, N.G., Caldwell, D.G., 2012. Statistical dynamical systems for skills acquisition in humanoids, in: Humanoid Robots (Humanoids), 2012 12th IEEE-RAS International Conference on, IEEE. pp. 323-329.

Catalano, J., Armstrong, T., Oates, T., 2006. Discovering patterns in real-valued time series, in: Knowledge Discovery in Databases: PKDD 2006, pp. 462 469.

Çeliktutan, O., Wolf, C., Sankur, B., Lombardi, E., 2012. Real-time exact graph matching with application in human action recognition, in: Human Behavior Understanding. Springer, pp. 17-28.
Chiu, B., Keogh, E., Lonardi, S., 2003. Probabilistic discovery of time series motifs, in: Proceedings of the ninth ACM SIGKDD international conference on Knowledge discovery and data mining, ACM, New York, NY, USA. pp. 493-498. doi:http://doi . acm.org/10.1145/956750.956808.

Chung, P.C., Liu, C.D., 2008. A daily behavior enabled hidden markov model for human behavior understanding. Pattern Recognition 41, 1572 - 1580. URL: http://www.sciencedirect.com/science/article/ $\mathrm{pii/S0031320307004700.}$

Gelper, S., Croux, C., 2007. Multivariate out-of-sample tests for granger causality. Comput. Stat. Data Anal. 51, 3319-3329.

Gombay, E., 2008. Change detection in autoregressive time series. J. Multivar. Anal. 99, 451-464. doi:http://dx.doi.org/10.1016/j.jmva. 2007. 01.003 .

Idé, T., Inoue, K., 2005. Knowledge discovery from heterogeneous dynamic systems using change-point correlations., in: SDM, SIAM. pp. 571-575.

Ijspeert, A.J., Nakanishi, J., Schaal, S., 2002. Learning attractor landscapes for learning motor primitives, in: Advances in neural information processing systems, pp. 1523-1530.

Jensen, K.L., Styczynxki, M.P., Rigoutsos, I., Stephanopoulos, G.N., 2006. A generic motif discovery algorithm for sequenctial data. BioInformatics 22, 21-28.

Keogh, E., Chu, S., Hart, D., Pazzani, M., 2001. An online algorithm for segmenting time series, in: Proceedings IEEE International Conference on Data Mining, 2001, pp. 289-296. URL: http://dx.doi.org/10.1109/ ICDM. 2001.989531, doi:10.1109/ICDM. 2001.989531.

Kulic, D., Takano, W., Nakamura, Y., 2007. Incremental on-line hierarchical clustering of whole body motion patterns. The 16th IEEE International Symposium on Robot and Human interactive Communication, 2007. , 10161021doi:10.1109/ROMAN. 2007.4415231.

Mohammad, Y., Nishida, T., 2009a. Constrained motif discovery in time series. New Generation Computing 27, 319-346.

Mohammad, Y., Nishida, T., 2009b. Robust singular spectrum transform, in: The 22nd International Conference on Industrial, Engineering and Other Applications of Applied Intelligent Systems IEA-AIE 2009, pp. 123-132.

Mohammad, Y., Nishida, T., 2010. Learning interaction protocols using augmented baysian networks applied to guided navigation, in: 2010 IEEE/RSJ International Conference on Intelligent Robots and Systems (IROS), pp. 4119 -4126. doi:10.1109/IROS . 2010.5651719.

Mohammad, Y., Nishida, T., 2014. Exact discovery of length-range motifs, in: Intelligent Information and Database Systems. Springer, pp. 23-32.

Mohammad, Y., Nishida, T., Okada, S., 2009. Unsupervised simultaneous learning of gestures, actions and their associations for human-robot interaction, in: Proceedings of the 2009 IEEE/RSJ international conference on Intelligent robots and systems, IEEE Press, Piscataway, NJ, USA. pp. $2537-$ 2544.

Mohammad, Y., Okada, S., Nishida, T., 2010. Autonomous development of gaze control for natural human-robot interaction, in: Proceedings of the 2010 workshop on Eye gaze in intelligent human machine interaction, ACM. pp. 63-70.

Mueen, A., 2013. Enumeration of time series motifs of all lengths, in: Data Mining (ICDM), 2013 IEEE 13th International Conference on, IEEE. pp. 547-556.

Mueen, A., Keogh, E.J., Zhu, Q., Cash, S., Westover, M.B., 2009. Exact discovery of time series motifs., in: SDM, pp. 473-484.

Oates, T., 2002. Peruse: An unsupervised algorithm for finding recurring patterns in time series, in: International Conference on Data Mining, pp. 330337.

Ohmura, R., Naya, F., Noma, H., Kogure, K., 2006. B-pack: a bluetoothbased wearable sensing device for nursing activity recognition, in: Wireless Pervasive Computing, 2006 1st International Symposium on, pp. 16-18. doi:10.1109/ISWPC. 2006.1613628.

Tee, K.P., Yan, R., Chua, Y., Huang, Z., 2010. Singularity-robust modular inverse kinematics for robotic gesture imitation, in: Robotics and Biomimetics (ROBIO), 2010 IEEE International Conference on, IEEE. pp. 920-925.

Tompa, M., Buhler, J., 2001. Finding motifs using random projections, in: 5th International Conference on Computational Molecular Biology, pp. 67-74. 